University of Windsor Scholarship at UWindsor

Civil and Environmental Engineering Publications

Department of Civil and Environmental

Engineering

$10-2014$

\title{
Detached eddy simulation of flow past an isolated inclined solar panel
}

\author{
M. Shademan \\ R. Balachandar \\ University of Windsor \\ R. M. Barron \\ University of Windsor
}

Follow this and additional works at: https://scholar.uwindsor.ca/civilengpub

\section{Recommended Citation}

Shademan, M.; Balachandar, R.; and Barron, R. M.. (2014). Detached eddy simulation of flow past an isolated inclined solar panel. Journal of Fluids and Structures, 50, 217-230.

https://scholar.uwindsor.ca/civilengpub/4 


\title{
Detached eddy simulation of flow past an isolated inclined solar panel
}

\author{
M. Shademan ${ }^{\mathrm{a}}$, R. Balachandar ${ }^{\mathrm{a}, \mathrm{b}}$, R.M. Barron ${ }^{\mathrm{a}, \mathrm{c}, *}$ \\ ${ }^{a}$ Department of Mechanical, Automotive E Materials Engineering, University of Windsor, Windsor, Ontario, Canada N9B 3P4 \\ ${ }^{\mathrm{b}}$ Department of Civil and Environmental Engineering, University of Windsor, Windsor, Ontario, Canada N9B 3P4 \\ ${ }^{\mathrm{c}}$ Department of Mathematics and Statistics, University of Windsor, Windsor, Ontario, Canada N9B 3P4
}

\section{A R T I C L E I N F O}

\section{Article history:}

Received 14 February 2014

Accepted 6 June 2014

Available online 14 August 2014

\section{Keywords:}

Detached eddy simulation

Solar panel

Ground clearance

Wind loading

\begin{abstract}
A B S T R A C T
Detached Eddy Simulations (DES) were carried out to analyze the influence of ground clearance and to estimate the unsteady wind loading on solar panels. A generic type of solar panel was considered at three different ground clearances. For the range of ground clearance values considered in the study, the results show that an increase in the clearance produces stronger vortex shedding fluctuations, higher shedding frequencies, and larger mean and unsteady wind loading. For the smallest ground clearance, an irregular vortex shedding was observed. The flow structures formed in the wake are analyzed using various indicators of turbulence characteristics including the vorticity and the $\lambda_{2}$ criterion. The flow structures suggest a complex formation and subsequent shedding of vortices. The absence of regular vortex shedding at the smallest ground clearance and the presence of the same at the mid-ground clearance value suggests a reference for minimizing the unsteady wind loading of solar panels. Furthermore, the unsteady results reveal that the lower sections of the panel are more vulnerable to wind loading.
\end{abstract}

(c) 2014 Elsevier Ltd. All rights reserved.

\section{Introduction}

Recently, there has been a proliferation in the use of solar panels for electricity generation. Unsteady wind loading on bluff bodies such as solar panels can cause significant damage to the structure and can have a significant influence on the life span of the structural elements. Several studies have been carried out to evaluate the wind loading on solar panels. Shademan and Hangan (2009, 2010) performed steady Reynolds-Averaged Navier-Stokes (RANS) simulations on flow past solar panels to analyze the effect of wind direction and panel inclination angle on the mean wind loading. They found the wind loading to be a maximum when the wind was from either the front or back face and that the corner panels may be subjected to increased loading. Bitsuamlak et al. (2010) carried out experiments and numerical simulations and noticed that the maximum wind loading occurred at a wind direction of $180^{\circ}$. Shademan et al. (2014) conducted steady RANS simulations to investigate the effect of gap spacing between panels on the mean wind loading. They found that an increase in the size of the gap significantly changed the flow structure behind the panels, which consequently affected the wind loading. Due to the pressure drop on the suction side in regions close to the gaps, these areas become more vulnerable to

\footnotetext{
* Corresponding author at: Department of Mathematics and Statistics, University of Windsor, Windsor, Ontario, Canada N9B 3P4.

Tel.: +1 519256 3000x3019; fax: +1519 9713649 .

E-mail address: az3@uwindsor.ca (R.M. Barron).
} 
higher wind loading. Although these studies provided information to better design solar panels, unsteady features were not considered. Unsteady features in the wake region are mainly caused by the generation and shedding of vortices. Since solar panels are installed at different ground clearances, the occurrence of the vortex shedding at a specific height can contribute significantly to the unsteady forces.

Literature shows that there have been wind tunnel studies to analyze the dependency of vortex shedding on ground clearance for other bluff bodies. For example, Wu and Martinuzzi (1997) carried out wind tunnel tests on a square cylinder and observed that the interaction between the upper and lower separating shear layers was reduced due to the weaker shear layer on the bottom of the cylinder. Durao et al. (1991) carried out laser Doppler measurements on a square cylinder at various heights above a plane channel wall. They noticed that regular vortex shedding was suppressed at a certain ground clearance.

Other studies have also shown the existence of a critical ground clearance for the generation of vortex shedding for different bluff bodies including circular cylinders (Bearman and Zdravkovich, 1978), triangular cylinders (Kamemoto et al., 1984), square cylinders (Taniguchi et al., 1983) and a two-dimensional normal plate (Everitt, 1982). Straatman and Martinuzzi (2002) performed RANS simulations of the experiments carried out by Wu and Martinuzzi (1997), using different second moment closure models. In their results, the computational prediction of drag appeared to be validated, but the lift and shedding frequency had major differences when compared to the experimental results. They suggested that the results can be improved by using Low-Re turbulence models.

Previous studies suggest that there is a strong relationship between vortex shedding and ground clearance. However, most of the numerical studies on flow past solar panels are RANS based which are incapable of capturing the vortex shedding (Shademan et al., 2014). To overcome this limitation, a Detached Eddy Simulation (DES) of flow past a generic set of solar panels was carried out in the current study. The recent results of Nasif et al. (2014) on DES for flow past bluff bodies makes this method promising for the current analysis. We investigate the effect of ground clearance on unsteady wind loading, pressure distribution, flow structures and evaluate a critical ground clearance at which vortex shedding begins. This critical ground clearance can be used as a reference for minimizing the unsteady wind loading on solar panels with similar aspect ratios.

\section{Geometry modeling}

The geometry for this study is shown in Fig. 1(a). The panel is $4.2 \mathrm{~m}$ in length, $3.2 \mathrm{~m}$ in width, $0.05 \mathrm{~m}$ thick, and set at an inclination angle $\varnothing=135^{\circ}$ with respect to the horizontal (Fig. 1(b)). This is a mid-range angle at which solar panels are generally installed. In this figure, $\Delta=W \sin (\pi-\varnothing)$ is the projected width of the panel. The origin of the coordinate system is located on the ground under the right bottom corner of the panel. Different ground clearance values $(H / \Delta=0.22,0.66$
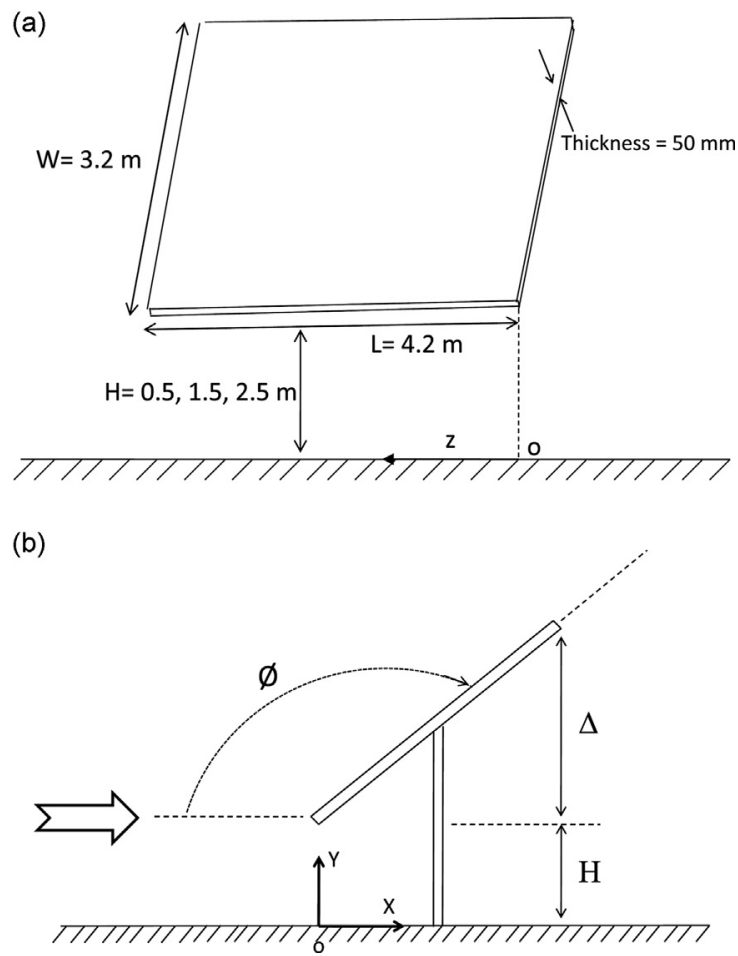

Fig. 1. (a) Geometry of the solar panel and (b) definition of inclination angle. 
and 1.11, where $H$ is the ground clearance) were modeled with the approach wind flow maintained parallel to the ground at zero yaw angle. This wind direction was selected based on the recommendation of Shademan and Hangan (2009, 2010) because of the potential for very high loading at this condition. Scaling the coordinates with $\Delta$, the panel is situated at $0 \leq x / \Delta \leq 1, H / \Delta \leq y \mid \Delta \leq 1+H / \Delta$ and $0 \leq z / \Delta \leq 1.85$. To ensure minimum influence of the computational boundaries, the domain is set to be $105 \mathrm{~m}$ long, $46 \mathrm{~m}$ wide and $20 \mathrm{~m}$ high (Fig. 2(a)). The blockage ratio is about $1.1 \%$, which is smaller than the 3\% upper limit recommended by Franke et al. (2007) to minimize wall interference effects.

A fully structured mesh of hexahedral elements is generated with a high-density mesh around the panel and on the ground to capture the high shear stresses in these regions. The surface grid spacing was maintained in the range of $h^{+}<1$, where $h^{+}=u_{\tau} h / \nu$ is a non-dimensional wall distance, $h$ is the normal distance from the wall, $\nu$ is the kinematic viscosity, $u_{\tau}=\left(\tau_{w} / \rho\right)^{0.5}$ is the friction velocity, $\tau_{w}$ is wall shear stress and $\rho$ is the density. Grid independence tests were performed by increasing the number of cells in $10 \%$ increments in each direction until there was no significant change in the aerodynamic forces on the panel. Based on these tests, a mesh with $220 \times 190 \times 125$ cells in $x, y$ and $z$ directions, respectively, was selected for subsequent simulations.

The side and top walls of the flow domain are considered as impermeable slip walls. The solar panel and ground are treated as no slip walls with zero roughness. A wind velocity profile representative of an atmospheric boundary layer is used at the inlet to the domain. Using ASCE 7-05 (2005), a wind velocity of $25 \mathrm{~m} / \mathrm{s}$ at the height of 10 m was selected. To simulate this inlet condition, a power-law equation is used

$$
\frac{u(y)}{U_{g}}=\left(\frac{y}{y_{g}}\right)^{\alpha},
$$

where $y_{g}$ is the atmospheric boundary layer height of the terrain, $U_{g}$ is the geostrophic wind velocity, $\alpha$ is an exponent dependent on the terrain and $y$ is the distance from the ground. In the current study an open terrain was considered with $\alpha=0.16$ and $y_{g}=300 \mathrm{~m}$. The inlet velocity profile was matched with the experimental data of ESDU 82026 (1982). Not considering the roughness effect on the ground results in inhomogeneity in the wind flow upstream of the panel. To keep the wind flow homogeneous while approaching the panel, the upstream length of the domain was set according to the recommendations of Blocken et al. (2007a, 2007b). Upstream velocity profiles including those at the inlet, approaching ( $10 \mathrm{~m}$ from the panel) and incident ( $2 \mathrm{~m}$ from the panel) locations are plotted in Fig. 2(b). In this figure, $H_{p}$ is the solar panel height and $U_{p}$ is the wind velocity at this level. This figure demonstrates that the change in the upstream profiles is insignificant and homogeneity of the upstream flow is preserved.

\section{Governing equations}

Detached Eddy Simulation (DES) is a hybrid method, which uses unsteady RANS models in the boundary layer and LES modeling in the free stream region. This method significantly reduces the computational costs compared to a conventional LES (Wang and Chen, 2009). In the present simulations, the SST $k-\omega$ RANS model (Menter, 1993) is used.

In addition to solving the momentum and continuity equations for velocity and pressure, the DES method calculates the turbulence from the following transport equations:

$$
\begin{aligned}
& \frac{\partial}{\partial t}(\rho k)+\frac{\partial}{\partial x_{i}}\left(\rho k u_{i}\right)=\frac{\partial}{\partial x_{j}}\left(\Gamma_{k} \frac{\partial k}{\partial x_{j}}\right)+G_{k}-Y_{k}+S_{k}, \\
& \frac{\partial}{\partial t}(\rho \omega)+\frac{\partial}{\partial x_{i}}\left(\rho \omega u_{i}\right)=\frac{\partial}{\partial x_{j}}\left(\Gamma_{\omega} \frac{\partial \omega}{\partial x_{j}}\right)+G_{\omega}-Y_{\omega}+S_{\omega} .
\end{aligned}
$$

(a)

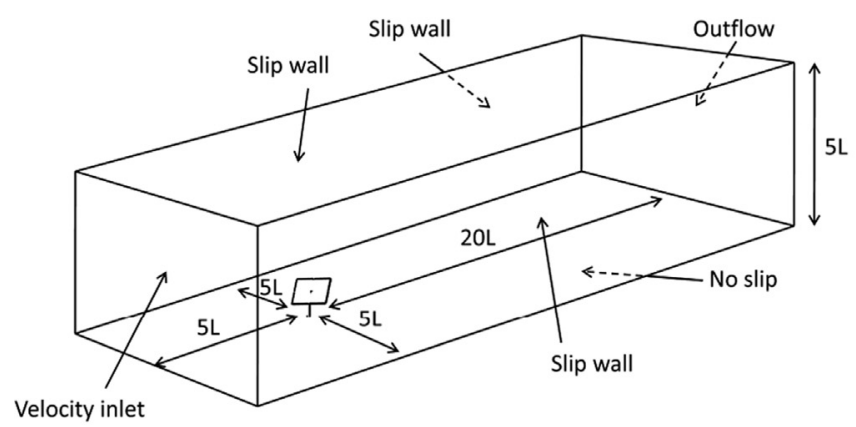

(b)

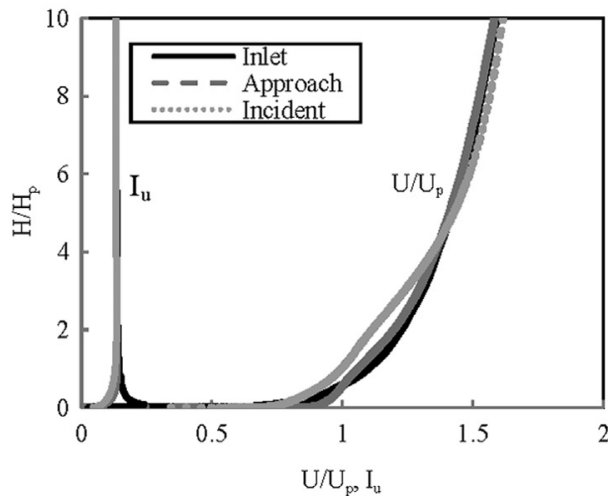

Fig. 2. (a) Domain dimensions and boundary conditions and (b) inlet, approach and incident velocity and turbulence intensity profiles. 
In these equations, $u_{i}$ is the velocity component in the $x_{i}(i=1,2,3)$ direction, $k$ is the turbulence kinetic energy, $\omega$ is the specific dissipation rate, $G_{k}$ represents the generation of $k$ due to mean velocity gradients, $G_{\omega}$ represents the generation of $\omega$, $\Gamma_{k}$ and $\Gamma_{\omega}$ represent the effective diffusivity of $k$ and $\omega, Y_{k}$ and $Y_{\omega}$ are the dissipation of $k$ and $\omega$ due to turbulence and $S_{k}$ and $S_{\omega}$ are user-defined source terms. In the SST $k-\omega$ based DES method, the dissipation term in Eq. (2) is written as

$$
Y_{k}=\rho \beta^{*} k \omega F_{D E S}
$$

where $\beta^{*}$ is a constant of the SST model and $F_{D E S}$ is determined from

$$
F_{D E S}=\max \left(\frac{L_{t}}{C_{D E S} \delta}\left(1-F_{S S T}\right), 1\right) \text { with } F_{S S T}=0, F_{1} \text { or } F_{2} \text {. }
$$

In this equation, $C_{D E S}$ is equal to 0.61 (Menter and Kuntz, 2003), $\delta$ is the maximum local grid spacing (i.e., maximum of $\delta x$, $\delta y, \delta z)$ and $L_{t}$ is the turbulent length scale, related to $k$ and $\omega$ by

$$
L_{t}=\frac{\sqrt{ } k}{\beta^{*} \omega}
$$

$F_{1}$ and $F_{2}$ are the blending functions of the SST model (Menter, 1993) and $F_{S S T}=0$ recovers the Strelets model (Strelets, 2001). These blending functions have a value of 1 in the near wall region and 0 away from the surface. Function $F_{1}$ is activated in the boundary layer while the function $F_{2}$ extends further out into the flow. Function $F_{1}$ is defined as

$$
F_{1}=\tanh \left(\Phi_{1}^{4}\right), \text { where } \Phi_{1}=\min \left[\max \left(\frac{\sqrt{k}}{0.09 \omega y}, \frac{500 \nu}{y^{2} \omega}\right), \frac{4 \rho k}{\sigma_{\omega, 2} D_{\omega}^{+} y^{2}}\right] .
$$

Here $\sigma_{\omega, 2}=1.168, y$ is the wall normal direction and $D_{\omega}^{+}$is the cross-diffusion term given by

$$
D_{\omega}^{+}=\max \left[2 \rho \frac{1}{\sigma_{\omega, 2}} \frac{1}{\omega} \frac{\partial k}{\partial x_{j}} \frac{\partial \omega}{\partial x_{j}}, 10^{-10}\right] .
$$

Function $F_{2}$ is defined as

$$
F_{2}=\tanh \left(\Phi_{2}^{2}\right), \text { where } \Phi_{2}=\max \left[2 \frac{\sqrt{k}}{0.09 \omega y}, \frac{500 \nu}{y^{2} \omega}\right] .
$$

The turbulent kinetic energy and dissipation rate applied at the inlet is based on the equations presented by Blocken et al. (2007a):

$$
\begin{aligned}
& k(y)=\frac{u_{A B L}^{* 2}}{\sqrt{C_{\mu}}}, \\
& \varepsilon(y)=\frac{u_{A B L}^{* 3}}{\kappa y},
\end{aligned}
$$

where $u_{A B L}^{*}$ is the atmospheric boundary layer friction velocity, $\kappa$ is the von Karman constant $(\approx 0.40-0.42)$ and $C_{\mu}$ is a model constant. In the case of the $k-\omega$ SST turbulence model, these profiles are combined to provide the specific dissipation rate $\omega\left(\omega=\varepsilon / C_{\mu} k\right)$. The turbulent kinetic energy profile, expressed in terms of turbulence intensity ( $\left.I_{u}\right)$, compares well with the values obtained from ESDU 83045 (1983).

The finite volume method is used to discretize the governing equations. The second-order upwind scheme is used to discretize the convective terms. Time-marching is performed using a fully-implicit second-order scheme. Based on the current mesh topology and in order to satisfy the Courant number condition, the time step is set at $1 \times 10^{-4}$. The SIMPLE

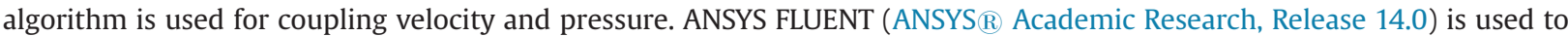
solve the governing equations. Mean values of flow variables are determined by averaging the instantaneous results long after the start of the simulation and the flow has reached a fully established condition.

\section{Validation}

To validate the numerical model, wind tunnel tests carried out by Fage and Johansen (1927) on flow past a flat plate at different inclination angles were modeled. They measured steady and unsteady flow quantities including mean pressure, shedding frequency, Strouhal number and aerodynamic loading.

Three-dimensional unsteady turbulent flow past an unconfined normal flat plate $\left(\varnothing=90^{\circ}\right)$ was computed using the DES model described in Section 3. The plate modeled in this simulation has a chord length of $0.15 \mathrm{~m}$ and a span of $1.5 \mathrm{~m}$. The recommendations of Bosch and Rodi (1996) were used to define the distance between the plate and boundaries in order to minimize blockage issues. The top, bottom and two side boundaries were considered to be walls with a slip condition, which is equivalent to assuming them to be far-field free stream-surfaces. No slip and no penetration boundary conditions were applied on the plate. A uniform velocity of $U_{\infty}=15 \mathrm{~m} / \mathrm{s}$ was set at the inlet. The corresponding Reynolds number based on the chord length is $10^{5}$. A fully structured hexahedral mesh was generated for the domain, which resulted in $5.9 \times 10^{6}$ 
cells. The mean pressure coefficient $\left(C_{p}=\left(P-P_{\infty}\right) /\left(0.5 \rho U_{\infty}^{2}\right)\right)$ obtained from the current simulations is presented in Fig. 3(a). Good agreement can be seen between the present simulations and the experimental results.

Fig. 3(b), which shows the iso-surfaces of pressure superimposed with the instantaneous static pressure contours $(x-y$ plane), clearly shows the vortex shedding. The Fast Fourier Transform of the pressure time history was carried out to compute the power spectra. The Strouhal number based on the peak frequency $(f)$, characteristic height $(\Delta)$ and approaching air velocity $\left(U_{\infty}\right)$ was calculated to be 0.140 (Fig. 3(c)), which matches well with the experimentally observed value of 0.147 . This validation provides guidance for the numerical setup and boundary conditions used for the simulation of the turbulent wind flow over a flat solar panel.

\section{Mean results for the solar panel}

The present DES modeling is designed to evaluate the unsteady wind forces caused by vortex shedding at different ground clearances $(H / \Delta=0.22,0.66$ and 1.11$)$. Determining a critical ground clearance at which the vortex shedding is likely to commence is of particular interest. Other flow parameters such as mean and turbulent quantities, force coefficients, pressure distribution and shedding frequency are also investigated.

\subsection{Mean flow and pressure fields}

Fig. 4 illustrates the mean flow sectional streamlines superimposed on mean pressure contours in two different $x-y$ planes, for $H / \Delta=0.22$. Fig. 4(a) corresponds to a plane near the mid-section $(z / \Delta=0.9)$, while Fig. 4(b) shows the results at a section close to either the left or right edge of the panel $(z \mid \Delta=0.05$ or 1.8). These figures demonstrate that the recirculation length $\left(L_{w}\right)$ decreases by moving from the mid-plane towards the outer edges of the panel. A similar reduction in the spread of the separation bubble occurs in the upper region of the wake, which is generated by the flow separating from the top edge.

In Fig. 4(a), a larger mean pressure difference between the windward and leeward surfaces can be seen in the region between the middle and the bottom sections of the panel, i.e., the stagnation zone is closer to the bottom edge of the panel. However, this pressure difference reduces from the middle towards the side edges, due to the decrease of pressure on the windward face. This is consistent with the reduction observed in the length of the recirculation bubble. According to the sectional streamlines and the pressure contours illustrated in Fig. 4(a) and (b), there is no significant difference in the vertical location of the core of the separation bubble when moving horizontally across the panel. However, in the streamwise direction the core of the vortex is closer to the panel in the two side planes $(z \mid \Delta=0.05,1.8)$ than in the middle section $(z \mid \Delta=0.9)$.

To get a better understanding of the flow field, several planes parallel to the ground and cutting through the panel were selected for a ground clearance of $H / \Delta=0.22$. Fig. 5(a) is representative of a plane close to the bottom edge of the panel

(a)

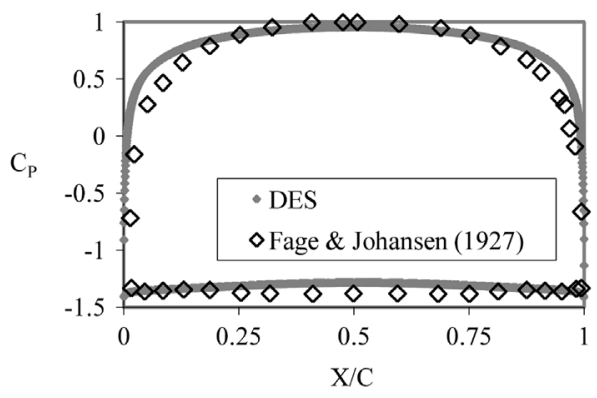

(c)

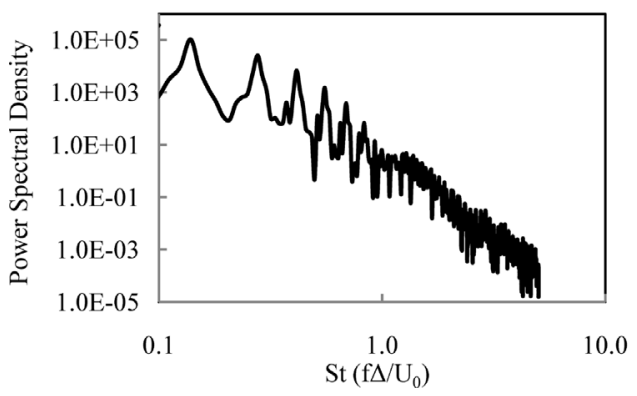

(b)

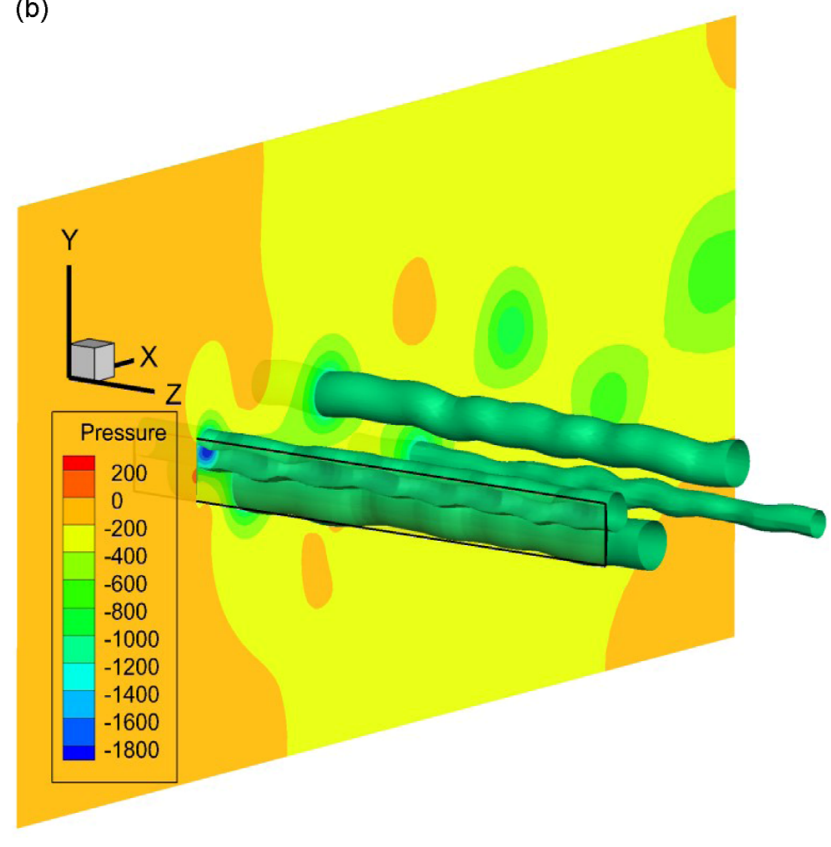

Fig. 3. (a) Mean $C_{p}$ comparison with experiments, (b) instantaneous pressure field (Pa) over flat plate $\left(\phi=90^{\circ}\right)$, and (c) power spectra density. 
(a)

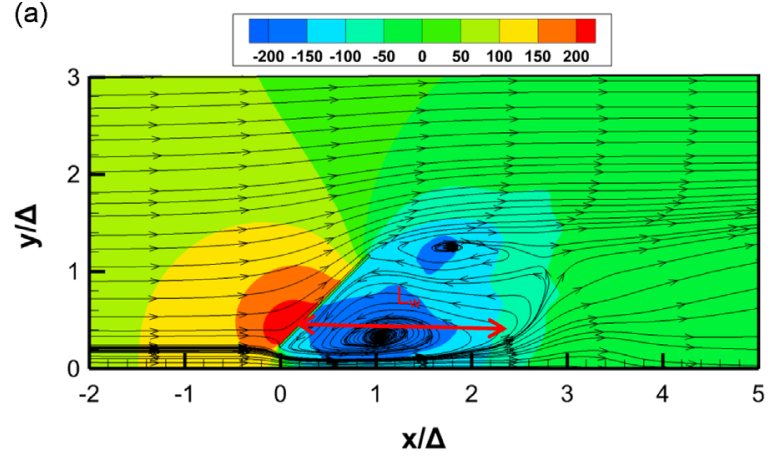

(b)

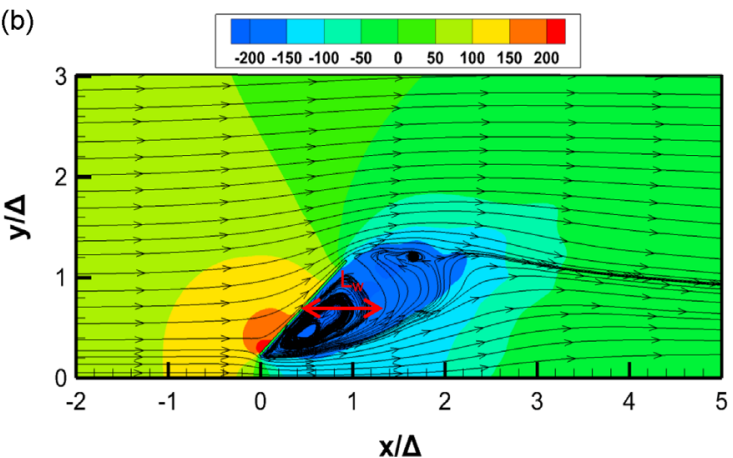

Fig. 4. $x-y$ plane sectional flow streamlines superimposed with mean pressure contours ( $\mathrm{Pa}$ ) for $H / \Delta=0.22$ : (a) mid-section and (b) side edge sections (right or left).

$(y / \Delta=0.3)$, while Fig. 5(b) and (c) show the results at the mid-section $(y / \Delta=0.7)$ and close to the top edge $(y / \Delta=1.1)$, respectively. At the bottom edge, no significant flow structure can be observed in the wake. As we move towards the midsection, two vortices appear which are generated by flow separating from the left and right edges. At the upper edge the two separation bubbles observed at $y / \Delta=0.7$ are slightly shifted downstream. From Figs. 4 and 5, three separation bubbles appear in the wake, one due to the separation of flow from the top edge and the other two from the two side edges of the panel. The mean sectional streamlines confirm the symmetrical behavior of the mean flow field about the $x-y$ mid-section $(z \mid \Delta=0.9)$. The role of ground friction and bottom edge separation will be discussed in detail in a later section.

In addition to the sectional streamlines, Fig. 5(a)-(c) also present the mean pressure contours. In the lower section (Fig. 5(a), $y / \Delta=0.3$ ), the high-pressure spots are distributed along the entire length of the panel. However, in the middle section (Fig. 5(b), $y \mid \Delta=0.7$ ), there is a region in the center of the panel that experiences higher pressures. At the top section (Fig. 5(c), $y \mid \Delta=1.1$ ), the pressure difference is much smaller than on the other sections. The maximum pressure difference between the windward and leeward surfaces is located between the bottom and middle planes of the panel. These locations on the solar panel can be considered as the regions where the mean aerodynamic loads are larger.

To track the flow structures observed in the $x-y$ and $x-z$ planes, cross-sections of the flow field were also analyzed in the $y-z$ plane. Fig. 6 shows results at $x / \Delta=1$ and 3. The two vortices generated from the two side edges of the panel are lifted up and merged as they move towards the downstream region, and the magnitude of the mean pressure reduces in the core of the vortices.

Fig. 7 presents the pressure and flow fields at the two other ground clearance values. At $H / \Delta=0.66$ (Fig. 7(a)), due to higher approaching wind velocities at this elevation, the magnitude of the pressure on the windward face is increased. Although the high-pressure region extends from the lower part of the panel towards the top edge, the length of the recirculation bubble is smaller compared to the flow at $H / \Delta=0.22$. Due to the increase of pressure in the windward region, the pressure difference between the panel faces increase. Increasing the ground clearance to $H / \Delta=1.11$ (Fig. 7(b)), results in a further reduction in the length of the recirculation bubble and the high-pressure region persists very close to the top edge. Consequently, the maximum mean pressure difference between the panel surfaces is larger in this case. Since the aerodynamic forces are a result of the pressure difference, it can be concluded that an increase in ground clearance leads to larger mean aerodynamic forces.

An important phenomenon from an engineering point of view is the positioning of the high- and low-pressure regions on the windward and leeward surfaces on the panel. Results (not presented in these figures) indicate that the location of the high-pressure region is almost fixed on the windward surface at a position close to the bottom edge of the panel. No movement of this region is observed during the shedding period. To the contrary, the low-pressure spots on the leeward face fluctuate with time, caused by the shedding of the vortices. As a consequence, the maximum pressure difference occurs 

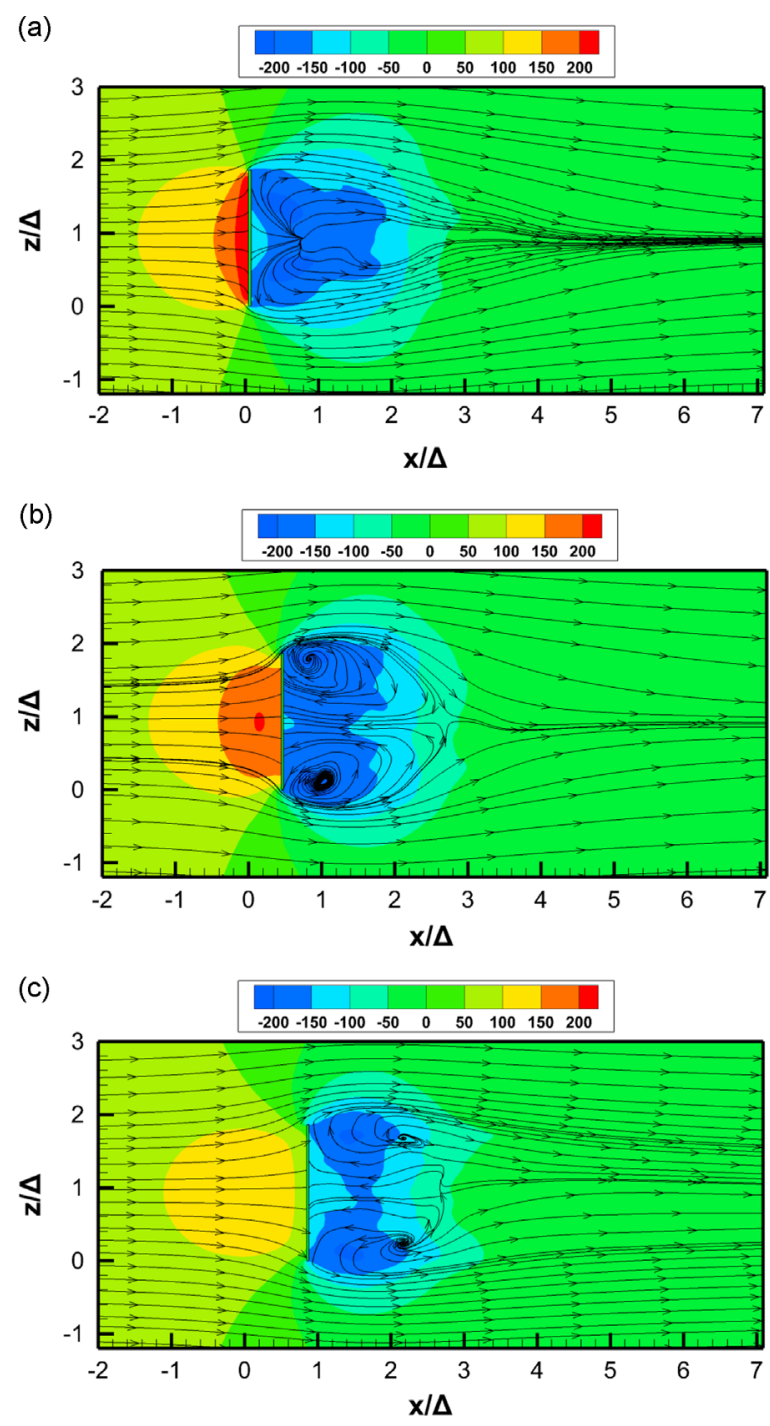

Fig. 5. $x-z$ plane sectional flow streamlines superimposed with mean pressure contours (Pa) for $H / \Delta=0.22$ : (a) $y / \Delta=0.3$, (b) $y / \Delta=0.7$, and (c) $y / \Delta=1.1$.

at the lower section of the panel close to the bottom edge. Therefore, special care has to be taken when designing the structure to minimize the aerodynamic fluctuations in this region.

Comparing Figs. 4(a), 7(a) and (b), it can be seen that increasing the ground clearance causes the size of this separation bubble at the top edge to decrease and at $H / \Delta=1.11$ it disappears. This likely occurs due to the larger flow rate passing under the panel through the gap region at higher ground clearances. By increasing the ground clearance to a certain level, the wake region is dominated by only two separation bubbles generated from the two side edges. It is interesting to note that ground clearance affects not only the flow near the bottom edge of the panel but also at the top edge.

\subsection{Flow structure in the gap region}

To investigate the influence of ground clearance on the mean flow structures in the gap region, mean vorticity contours at the mid-section $(z \mid \Delta=0.9)$ and mean wall shear stress on the ground are plotted. At $H / \Delta=0.22$ (Fig. 8(a)), the shear flow which separates from the bottom edge interacts with the ground and is demarcated by two regions $(x / \Delta<1$ and $1<x / \Delta<2)$. By increasing the ground clearance to $H / \Delta=0.66$ (Fig. 8(b)), the bottom shear flow finds enough space to escape through the gap as the high vorticity contours do not touch the ground. Further increase of the ground clearance to $H / \Delta=1.11$ (Fig. 8(c)) allows for a similar penetration of the bottom flow into the downstream region.

Fig. 8(d)-(f) provide a measure of the wall shear stress (bed friction) generated by the flow passing through the gap region. As Fig. 8(d) demonstrates, for $H / \Delta=0.22$ the high bed friction regions are located below the bottom and at the left and right edges of the panel. The high bed friction region destabilizes the flow separating from the bottom edge, causing 

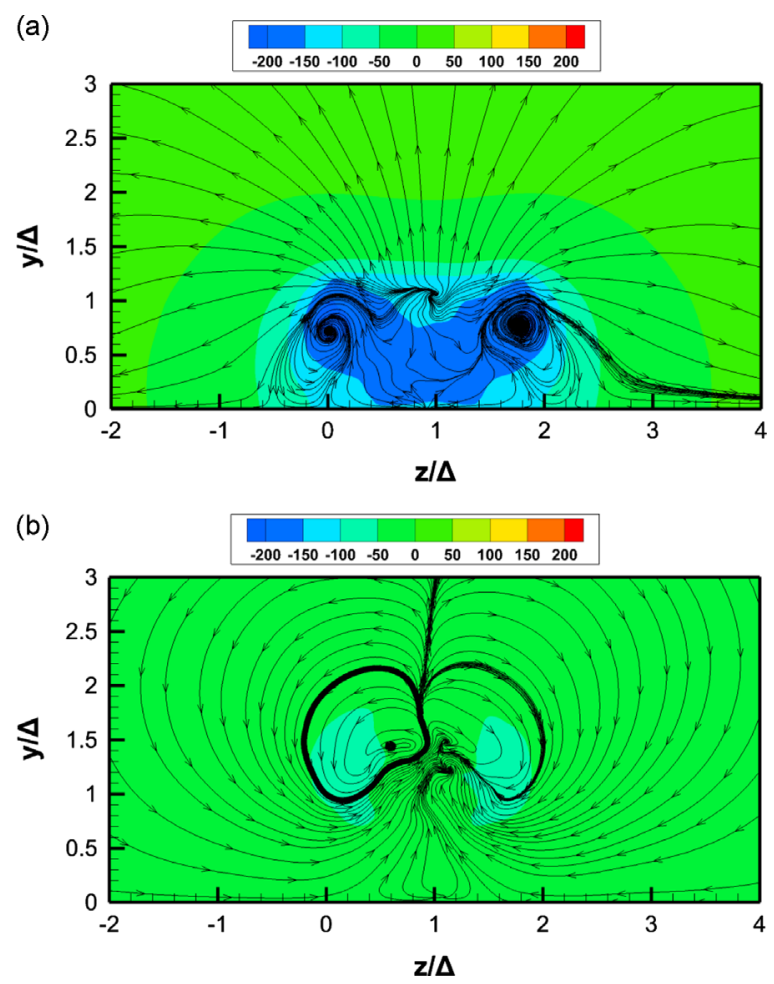

Fig. 6. $y-z$ plane sectional flow streamlines superimposed with mean pressure contours (Pa) for $H / \Delta=0.22$ : (a) $x / \Delta=1$ and (b) $x / \Delta=3$.
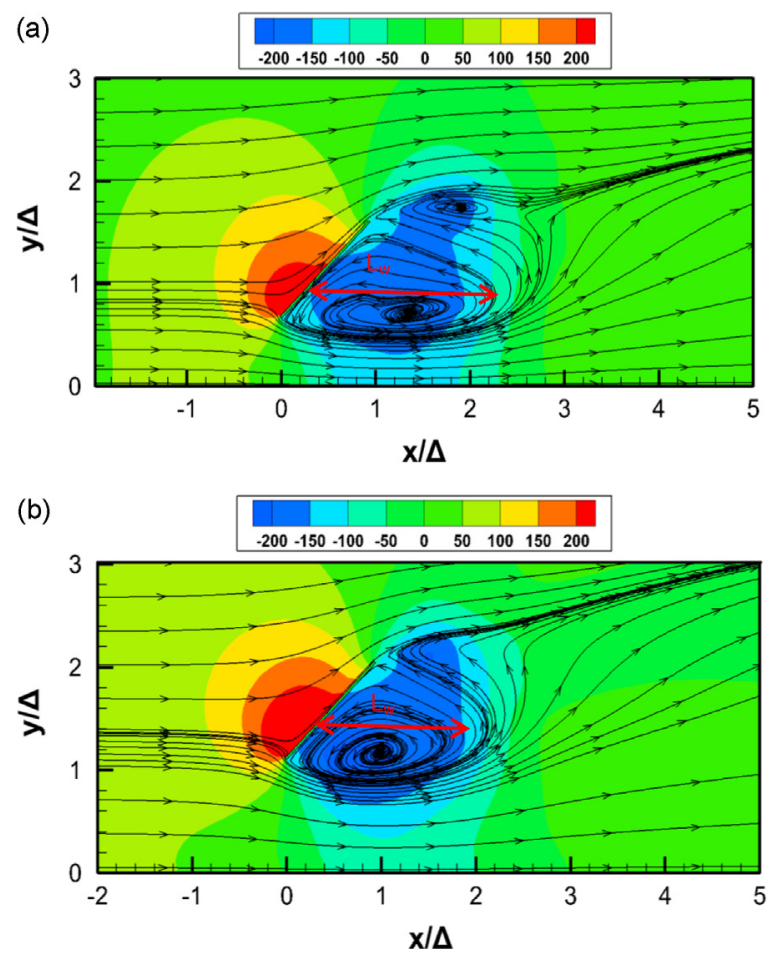

Fig. 7. Sectional flow streamlines in streamwise direction superimposed with mean pressure contours (Pa): (a) $H / \Delta=0.66$ and (b) $H / \Delta=1.11$.

high vorticity spots between $0<x / \Delta<2$ and across the entire length of the panel. A reduction in the magnitude of the ground bed friction is observed when the ground clearance increases. Higher friction is associated with more blockage and ground interference, which causes disruption of the vorticity contours in the wake. For larger ground clearances, lower bed friction provides easier passage of the flow through the gap and deeper penetration into the downstream region. 

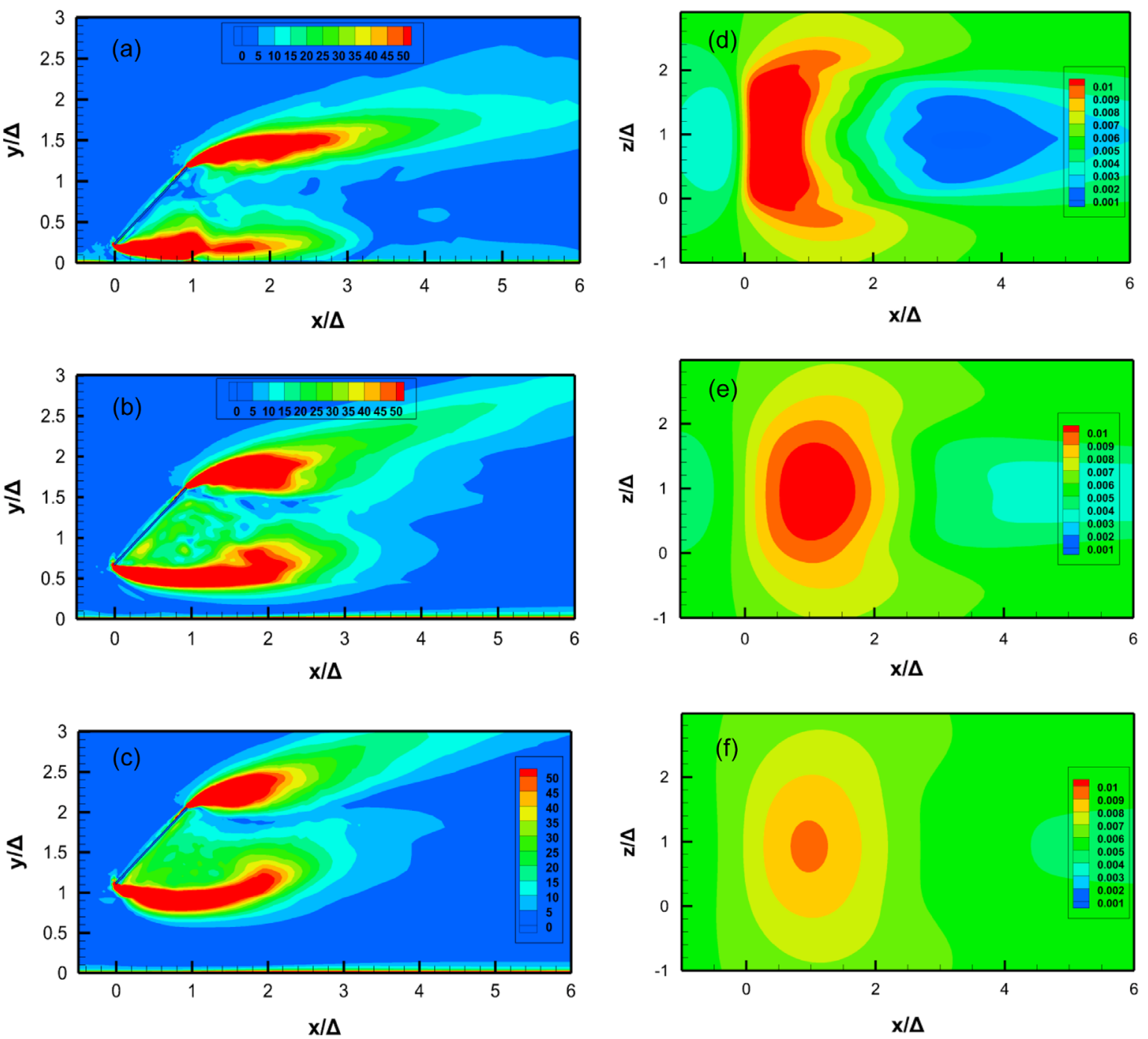

Fig. 8. Mean vorticity contours (a-c) on mid-section $(z / \Delta=0.9)$ and ground shear stress contours (d-f): (a, d) $H / \Delta=0.22$, (b, e) $H / \Delta=0.66$, and (c, f) $H / \Delta=1.11$.

\section{Unsteady results for the solar panel}

\subsection{Forces and shedding frequency}

Fig. 9 shows the time history of lift coefficients $\left(C_{L}=F_{L} /\left(0.5 \rho U_{0}^{2}\right)\right)$ for each $H / \Delta$ where $F_{L}$ is the lift force, $\rho$ is the density and $U_{0}$ is the averaged streamwise velocity. At $H / \Delta=0.22$, the minimum and maximum $C_{L}$ peaks fluctuate between -0.60 and -0.65 . At $H / \Delta=0.66$, the magnitude of the lift coefficient increases due to the higher approaching wind velocity and resulting pressure difference. The minimum and maximum peaks for the lift coefficient are -0.67 and -0.7 . At $H / \Delta=1.11$, the lift coefficient varies between -0.9 and -0.97 which is significantly larger in magnitude than the other two cases.

Increase of the ground clearance from $H / \Delta=0.22$ to 1.11 results in higher mean and unsteady wind loading on the structure. A maximum increase of $10 \%$ is observed in the unsteady component of the lift force. Another important observation is the increase in the amplitude of the fluctuating force when the ground clearance increases. This has to be taken into consideration since the large amplitude oscillations can cause fatigue issues leading to failure of the structure.

To investigate the shedding frequency of the vortices generated by the interaction of the wind flow with the panel, pressure was monitored at a point in the wake. Fig. 10 shows the variation of the power spectral density with Strouhal number $\left(S t=f \Delta / U_{0}\right)$, where $f$ is the frequency of vortex shedding. The Strouhal number calculated at $H / \Delta=0.22$ is 0.139 , however it increases to 0.167 for $H / \Delta=0.66$ and to 0.179 for $H / \Delta=1.11$. The results presented in Fig. 10 also show the increase in the peak of the power spectral density as a consequence of increasing the ground clearance. 


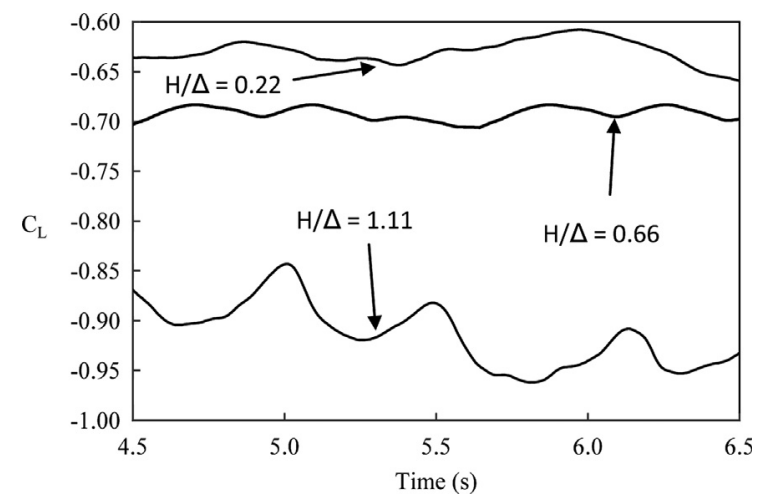

Fig. 9. Time history of the lift coefficient: (a) $H / \Delta=0.22$, (b) $H / \Delta=0.66$, and (c) $H / \Delta=1.11$.

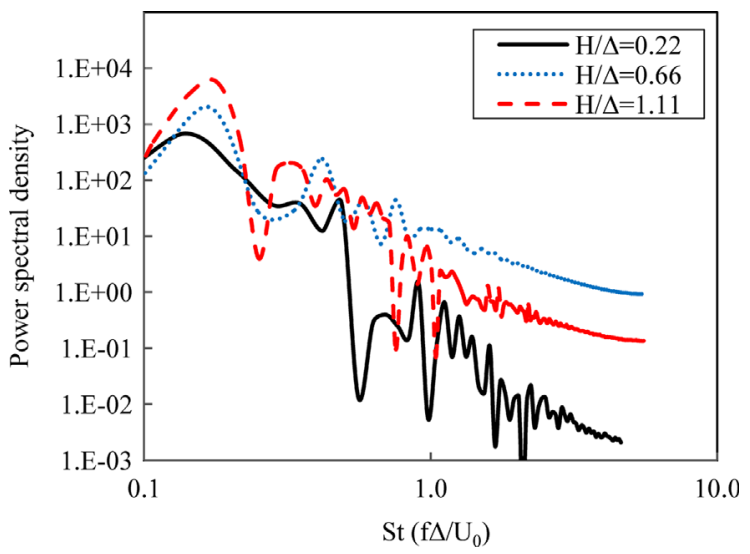

Fig. 10. Variation of Strouhal number with $H / \Delta$.

\subsection{Flow structure}

Forces and Strouhal number do not provide much information about the unsteady phenomena occurring in the flow field. The structure of the time-dependent flow field can be elucidated by examining the instantaneous vorticity.

The vorticity magnitude contours for a complete shedding period ( $T=0.81 \mathrm{~s}$ ) at equal time intervals of $T / 3$ are shown in Fig. 11(a)-(d) (left column) in the $x-y$ mid-section plane $(z \mid \Delta=0.9)$, for $H / \Delta=0.22$. The contours show several high-vorticity regions behind the panel, including the shear flows separating from the top and bottom edges of the panel. At this ground clearance, the high-vorticity regions emanating from the gap are in direct contact with the ground and consequently their evolution and movement has been strongly influenced by the bed friction. Additionally, a number of high-vorticity patches (marked as A-C) can be identified on the ground. To identify the source of these small patches, Fig. 11(e)-(h) (right column) are plotted showing the vorticity contours in the $x-z$ plane at the near-ground height of $y / \Delta=0.05$. Close examination indicates that these patches are part of the shear flows formed from the left and right edges of the panel, and appear to meander as they migrate downstream.

Fig. 12 shows the vorticity contours corresponding to the ground clearance of $H \mid \Delta=0.66$, on the same planes as in Fig. 11 . The contours at the highest ground clearance are similar to those seen in Fig. 12 and are not presented here. The highvorticity regions located at the top and bottom edges of the panel are swept downstream as time advances (left column). Fig. 12(e)-(h) show that the left and right shear flows do not leave an imprint on the ground. The structures that were observed in the flow at $H / \Delta=0.22$, i.e., patches such as A-C (Fig. 11), are absent at the larger ground clearance.

Fig. 13 shows the instantaneous vorticity magnitude contours in a horizontal plane passing through the bottom edge of the panel (i.e., $y=H$ ) in the $x-z$ plane. Fig. 13(a) shows that when the panel is close to the ground, the vortices retain their strength and travel downstream, meandering around the mid-section. At the higher ground clearances of $H / \Delta=0.66$ (Fig. 13 (b)) and $H / \Delta=1.11$ (Fig. 13(c)), the shed vortices take on a symmetrical behavior about the mid-section and since they are swept upward, lose their strength (in this plane) within a short distance behind the panel.

To obtain a three-dimensional perspective of the instantaneous flow structures, the $\lambda_{2}$ criterion developed by Jeong and Hussain (1995) is adopted in the current study. This method identifies the core of the vortices that exist in the flow field, using the fact that these cores are related to the locations of minimum pressure in the flow. They derived the $\lambda_{2}$ criterion by taking the gradient of the Navier-Stokes equation and decomposing the acceleration gradient term into symmetric and antisymmetric parts, expressed as $S_{i j} S_{i j}+\Omega_{i j} \Omega_{i j}$, where $S_{i j}$ and $\Omega_{i j}$ are the symmetric and antisymmetric parts of the velocity 

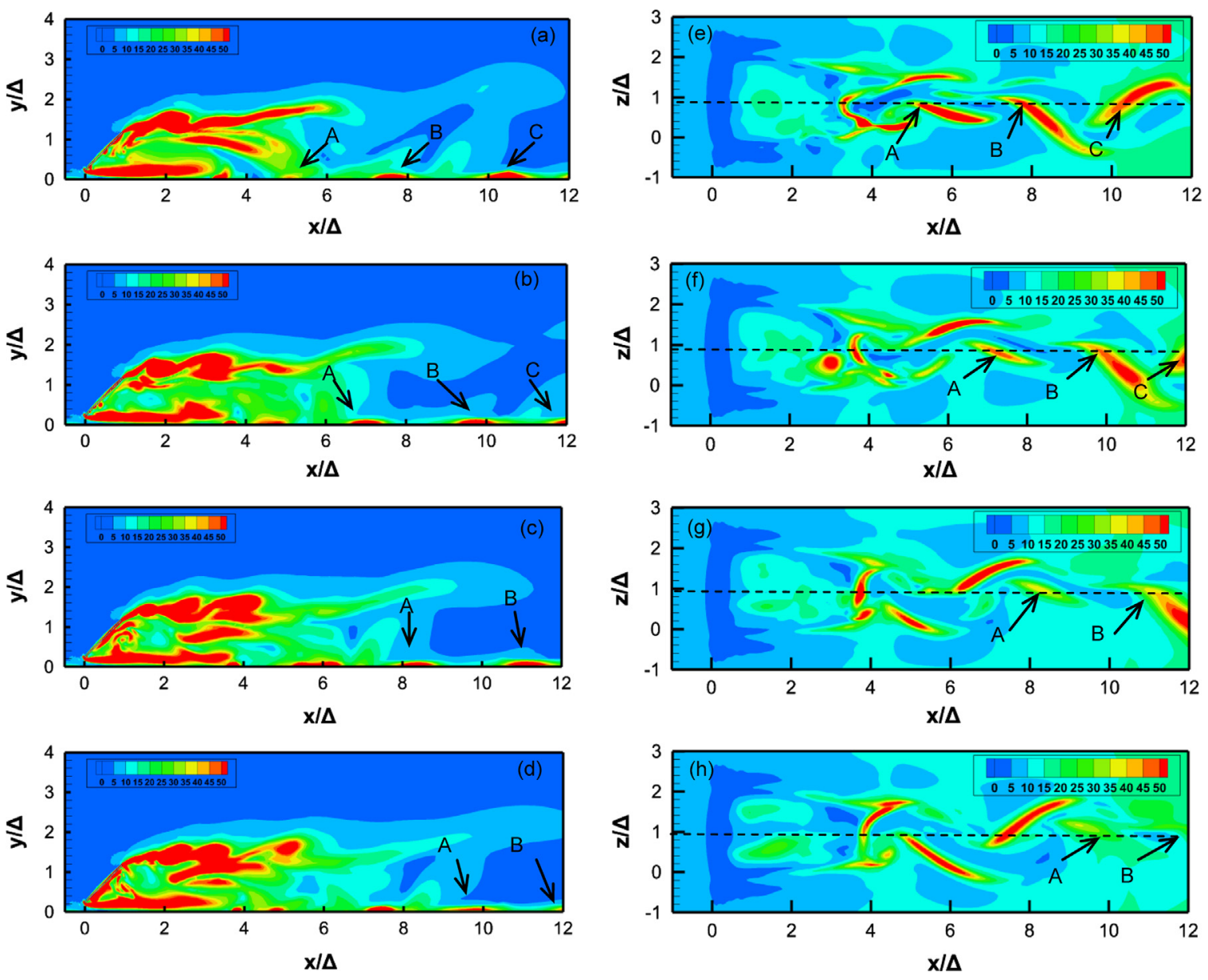

Fig. 11. Vorticity magnitude contours at equal time intervals for one shedding period, $(H / \Delta=0.22):(\mathrm{a}-\mathrm{d}) z / \Delta=0.9$ and $(\mathrm{e}-\mathrm{h}) y / \Delta=0.05$.

gradient tensor, respectively. The Hessian of the pressure can then be connected to the vortical motions in the flow. The $S_{i j} S_{i j}+\Omega_{i j} \Omega_{i j}$ tensor has three real eigenvalues $\lambda_{1} \geq \lambda_{2} \geq \lambda_{3}$. The point of minimum local pressure requires two eigenvalues of this tensor to be negative. The second largest eigenvalue $\lambda_{2}$ is representative of the local pressure minima region. Thus the iso-surfaces of $\lambda_{2}$ can be used to visualize instantaneous structures in the flow.

The $\lambda_{2}$ criteria is used to provide a sample of the instantaneous flow field in Fig. 14, illustrating the separation, displacement and expansion of the vortices, up to a streamwise distance of $x / \Delta \sim 20$. The color on the vortices represents the magnitude of streamwise velocity. In all cases, hairpin-like vortices form in the wake. Due to prevailing velocities in the upper regions of wake, these vortices are stretched and expanded with increasing downstream distance. While near the ground, at $H / \Delta=0.22$, Fig. 14(a)-(c) show that the two legs of the hairpin vortices are distorted over one shedding period due to the interaction with the ground. This is consistent with the phenomenon observed in Fig. 11(e)-(h) where small patches of high vorticity were observed.

Fig. 14(d)-(f) compare the vortices for different ground clearances. At $H / \Delta=0.66$ (Fig. 14(e)), the flow finds enough space to escape freely from the bottom edge and consequently the hairpin vortices retain their complete form with their two legs intact, unlike the $H / \Delta=0.22$ case (Fig. 14(d)). This behavior is consistent with results presented in Fig. 12(e)-(h), which illustrate high-vorticity patches due to the cross-sectional plane cutting through the bottom of the hairpin vortices. Fig. 14(e) and (f) indicate that the shed vortices maintain their structure at higher ground clearances, while expanding and stretching with increasing downstream distance. As these vortices travel downstream they appear to bend towards the ground and their legs get stretched. However, since the hairpin legs are not attached to the ground, these vortices are more stable than those generated at smaller clearances.

\section{Conclusions}

Detached Eddy Simulation was carried out to investigate the effect of ground clearance on mean and fluctuating wind loads experienced by a solar panel. The DES method was used to predict the vortex shedding, which could not be captured in RANS simulations. 

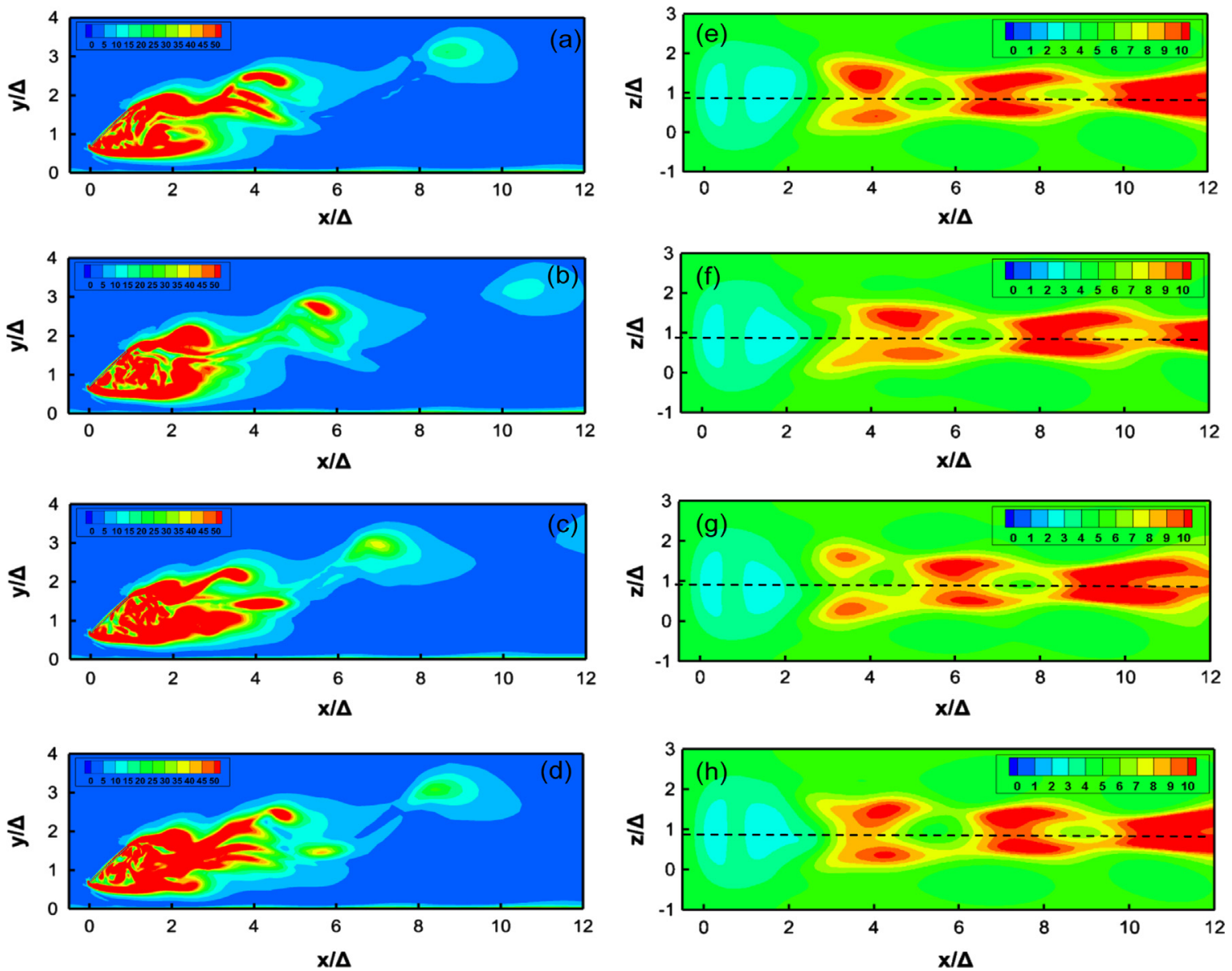

Fig. 12. Vorticity magnitude contours at equal time intervals $(T / 3)$ for one shedding period for $H / \Delta=0.66$ : $($ a-d) $z / \Delta=0.9$ and (e-h) $y / \Delta=0.05$.
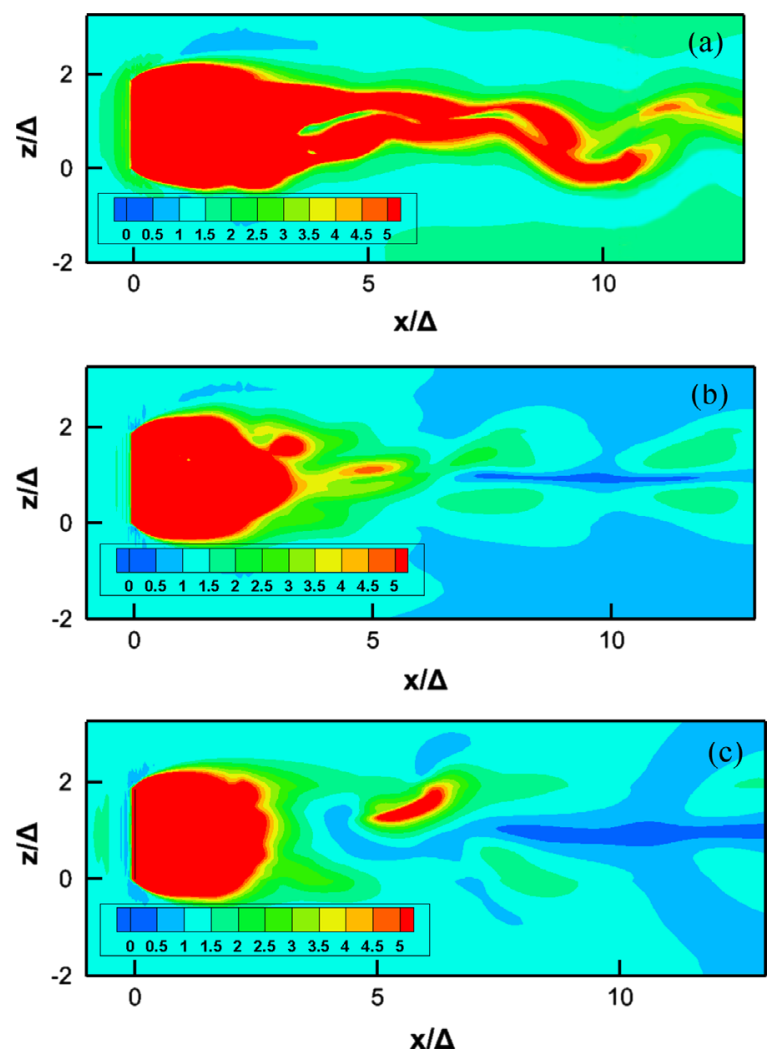

Fig. 13. $x-z$ plane instantaneous vorticity magnitude contours at $y=H$ : (a) $H / \Delta=0.22$, (b) $H / \Delta=0.66$, and (c) $H / \Delta=1.11$. 
(a)

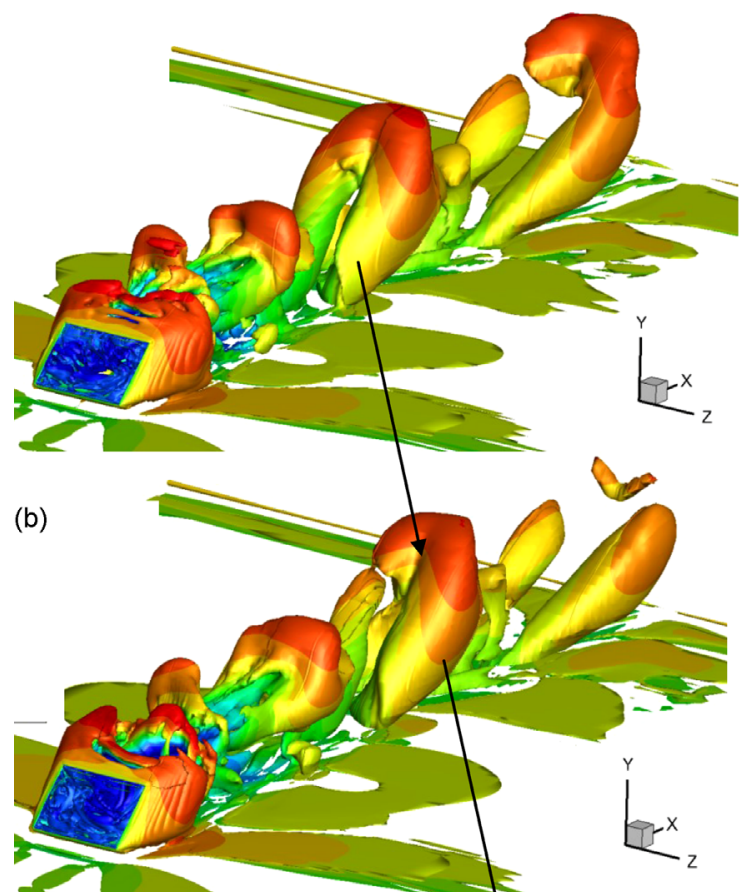

(c)

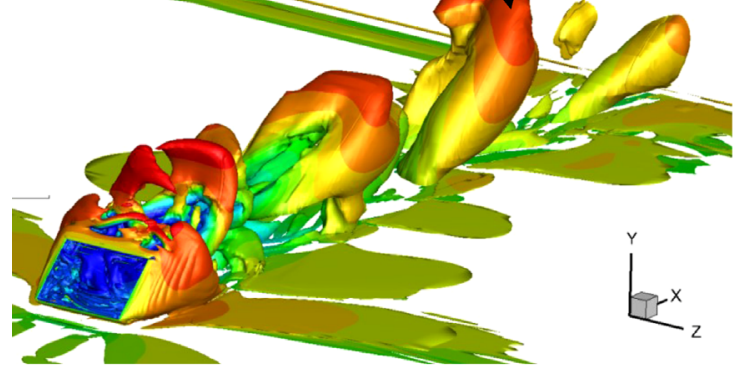

(d)

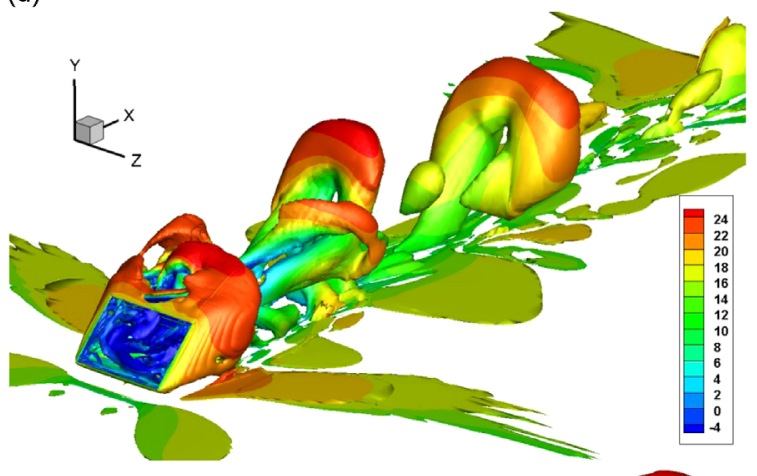

(e)
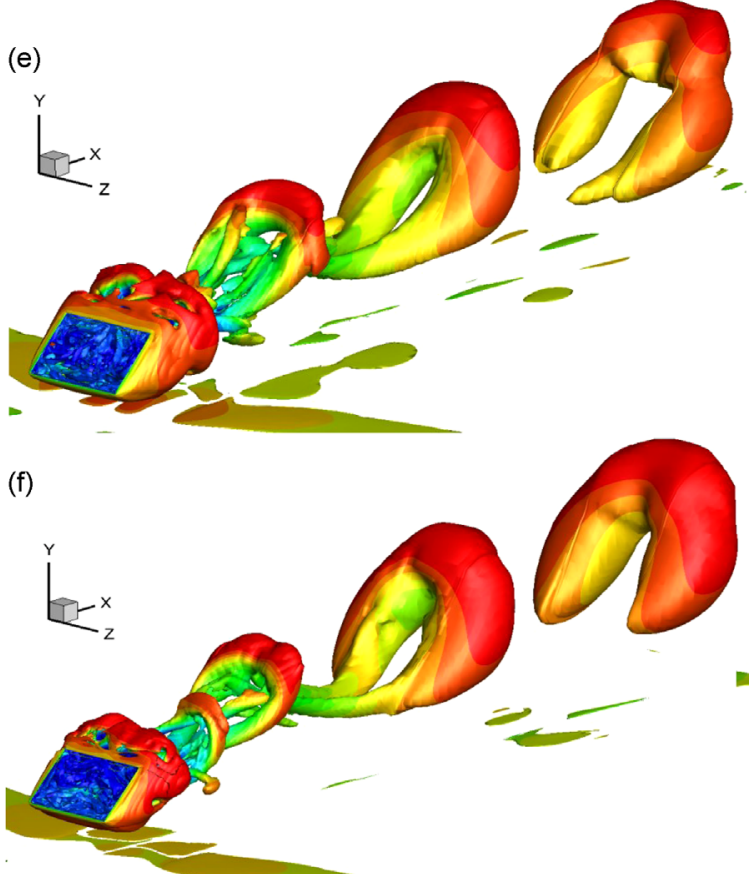

Fig. 14. Instantaneous $\lambda_{2}$ contours (3D view): (a-c) for one shedding period at equal time intervals of $T / 4(H / \Delta=0.22)$, (d) $H / \Delta=0.22$, (e) $H / \Delta=0.66$ and (f) $H / \Delta=1.11$.

It has been shown that the mean flow field past a solar panel is significantly dependent on ground clearance. An increase in ground clearance increases the stagnation pressure and the mean pressure difference between the surfaces of the panel, leading to a larger mean wind loading.

Three different mean flow structures are observed in the wake region for the smallest ground clearance case. Two structures are the result of the separation of flow from the left and right edges and one is due to the separation of flow from the top edge. As the ground clearance increases, the top separation bubble loses its strength and, at the highest ground clearance, this separation bubble disappears.

Analysis indicates that increase in ground clearance leads to stronger vortex shedding and larger unsteady forces on the panel. At small ground clearance, the separated flows from both left and right edges touch the ground and leave their imprint. However, this behavior is weakened or completely suppressed at larger ground clearances.

Analysis of the unsteady flow structures using the $\lambda_{2}$ criterion reveals the generation of hairpin-like vortices which stretched and expanded while moving downstream. At the smallest ground clearance the distorted shape of the hairpin vortices is due to the small gap, which weakens the separated flow from the bottom edge. These vortices are highly unstable and break up within a short distance behind the panel. Furthermore, the fluctuation of the vortices on the leeward face makes the lower section of the panel very critical in terms of wind loading. At larger ground clearance the air finds enough space to separate from the bottom edge, allowing the hairpin vortices to maintain their structure. Based on these observations the critical ground clearance above which the vortex shedding takes a regular form, which should be avoided, occurs at a level between the smallest and middle ground clearances simulated in this study, and flat solar panels should be installed close to the ground level to minimize the long term effects of the aerodynamic forces of the panels. 


\section{Acknowledgment}

This research was supported by NSERC of Canada through the Vanier Canada Graduate Scholarship program. Facilities of the Shared Hierarchical Academic Research Computing Network (SHARCNET) were used for conducting the simulations.

\section{References}

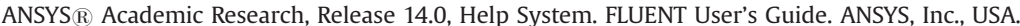

ASCE 7-05, 2005. Minimum Design Loads for Buildings and Other Structures. American Society of Civil Engineers, ASCE, New York, USA.

Bearman, P.W., Zdravkovich, M.M., 1978. Flow around a circular cylinder near a plane boundary. Journal of Fluid Mechanics 89, 33-50.

Bitsuamlak, G.T., Dagnew, A.K., Erwin, J., 2010. Evaluation of wind loads on solar panel modules using CFD. In: Proceedings of the 5th International Symposium on Computational Wind Engineering (CWE2010), Chapel Hill, NC, USA.

Blocken, B., Carmeliet, J., Stathopoulos, T., 2007a. CFD evaluation of the wind speed conditions in passages between buildings: effect of wall-function roughness modifications on the atmospheric boundary layer flow. Journal of Wind Engineering and Industrial Aerodynamics 95 (9-11), 941-962.

Blocken, B., Stathopoulos, T., Carmeliet, J., 2007b. CFD simulation of the atmospheric boundary layer: wall function problems. Atmospheric Environment 41 (2), 238-252.

Bosch, G., Rodi, W., 1996. Simulation of vortex shedding past a square cylinder near a wall. International Journal of Heat and Fluid Flow 17 (3), $267-275$.

Durao, D.F.G., Gouveia, P.S.T., Pereira, J.C.F., 1991. Velocity characteristics of the flow around a square cross-section cylinder placed near a channel wall. Experiments in Fluids 11, 298-304.

ESDU, 1982. Strong Winds in the Atmospheric Boundary Layer, Part 1: Mean-Hourly Wind Speeds. Engineering Science Data Unit Number 82026.

ESDU, 1983. Strong Winds in the Atmospheric Boundary Layer, Part 2: Discrete Gust Speeds. Engineering Science Data Unit Number 83045.

Everitt, K.W., 1982. A normal flat plate close to a large plane surface. Aeronautical Quarterly 33, 90-103.

Fage, A., Johansen, F.C., 1927. On the flow of air behind an inclined flat plate of infinite span. Proceedings of the Royal Society of London Series A 116 $170-197$.

Franke, J., Hellsten, A., Schlünzen, H., Carissimo, B., 2007. Best Practice Guideline for the CFD Simulation of Flows in the Urban Environment. COST Action 732, Quality Assurance and Improvement of Micro-Scale Meteorological Models.

Jeong, J., Hussain, F., 1995. On the identification of a vortex. Journal of Fluid Mechanics 285, 69-94.

Kamemoto, K., Oda, Y., Aizawa, M., 1984. Characteristics of the flow around a bluff body near a plane surface. Bulletin of the Japan Society of Mechanica Engineering 27, 1637-1643.

Menter, F.R., 1993. Zonal two-equation $k-\omega$ turbulence models for aerodynamic flows. AIAA Paper 1993-2906, In: Proceedings of AIAA 24th Fluid Dynamics Conference, Orlando, FL.

Menter, F.R., Kuntz, M., 2003. A zonal SST-DES formulation. In: DES Workshop, Technical University, St. Petersburg, Russia.

Nasif, G., Barron, R.M., Balachandar, R., 2014. DES evaluation of near-wake characteristics in a shallow flow. Journal of Fluid \& Structures 45, $153-163$.

Shademan, M., Barron, R.M., Balachandar, R., Hangan, H., 2014. Numerical simulation of wind loading on ground-mounted solar panels at different flow configurations. Canadian Journal of Civil Engineering. http://dx.doi.org/10.1139/cjce-2013-0537, in press.

Shademan, M., Hangan, H., 2009. Wind loading on solar panels at different inclination angles. In: Proceedings of the 11th Conference of American Society of Wind Engineers, San Juan, Puerto Rico.

Shademan, M., Hangan, H., 2010. Wind loading on solar panels at different azimuthal and inclination angles. In: Proceedings of the 5th International Symposium on Computational Wind Engineering (CWE2010), Chapel Hill, NC, USA.

Straatman, A.G., Martinuzzi, R.J., 2002. A comparison of second-moment closure models in the prediction of vortex shedding from a square cylinder near a wall. Journal of Fluids Engineering 124, 728-736.

Strelets, M., 2001. Detached eddy simulation of massively separated flows. AIAA Paper 2001-0879. In: Proceedings of the 39th AIAA Aerospace Sciences Meeting and Exhibit, Reno, NV.

Taniguchi, S., Miyakoshi, K., Dohda, S., 1983. Interference between plane wall and two-dimensional rectangular cylinder. Transaction of Japanese Society of Mechanical Engineering 49, 2522-2529.

Wang, M., Chen, Q., 2009. Assessment of various turbulence models for transitional flows in enclosed environment. HVAC\&R Research 15 (6), 1099-1119.

Wu, K.C.Q., Martinuzzi, R.J., 1997. Experimental study of the turbulent wake flow behind a square cylinder near a wall. Paper FEDSM97-3151. In: Proceedings of ASME FED Summer Meeting, Vancouver, BC. 